\title{
Seasonal Incidence of Spider Mite (Tetranychus urticae Koch) (Acari: Tetranychidae) Infesting Rose under Poly House Condition
}

\author{
S.R. Desai*, K.G. Patel and Abhishek Shukla \\ Department of Agricultural Entomology, N. M. College of Agriculture, Navsari Agricultural \\ University, Navsari 396 450, Gujarat, India \\ *Corresponding author
}

\section{A B S T R A C T}

\begin{tabular}{|l|}
\hline Ke y w or d s \\
Seasonal incidence, \\
Spider mite, Rose, \\
Tetranychus urticae
\end{tabular}

Study on seasonal incidence on spider mite (Tetranychus urticae Koch) infesting rose under poly house condition were carried out at Navsari Agricultural University, Navsari, Gujarat during 2015-2016. Under the present study the two spotted spider mite (T. urticae) was recorded on rose (cv. Top secret) grown under the poly house condition. Seasonal incidence of $T$. urticae revealed that, the spider mite population observed from $3^{\text {rd }}$ week of July to $1^{\text {st }}$ week of August. It was highly significant correlation with minimum temperature and average temperature, while negative nonsignificant correlation with average relative humidity and maximum temperature.

\section{Introduction}

Flower, the most beautiful and fascinating part of nature, have power to overwhelm anybody's heart with love, happiness and joy. The area under floricultural crops in India is $2,42,710$ hectares with production of 15,45,250 Metric tonnes. As far as Gujarat state is concerned the area under flower production is 19,670 ha. India produced 6,91,190 Metric tonnes cut flowers during the year 2016. (Anonymous, 2016). Rose has been the world's most favorite and unchallenged flower and reigned supreme as the "Queen of flowers". A title bestowed on it by the Greek poetess "Sappho" and none has since questioned its right to the title. Rose plant is attacked by number of insect pests like bud borers, thrips, scales, aphids, weevils, chafer beetles, leaf eating caterpillars and non-insect pest like red spider mite. Among the non-insect pests the two spotted red spider mite, Tetranychus urticae Koch (Acari: Tetranychidae) is the most important one causing serious damage to rose and other crops grown in poly house as well as in open field.

Today, it poses greatest threat to poly house roses. They usually colonize on under surface of leaves and when population is very high, they can effect on all parts of the plant including flowers. They prefer nitrogen rich young leaves, but in well-established colonies 
older leaves become heavily infected. As, the population build up, spider mites usually spine sufficient webbing to cover the entire plant. The spider mite pierces the epidermis cells of the host plant with its stylet like mouthparts known as cheliceri. To develop effective management strategies against any pest it is very important to understand its seasonal activities, therefore an experiment was undertaken to investigate the seasonal activities of $T$. urticae on poly house rose.

\section{Materials and Methods}

The study on seasonal incidence of spider mite, $T$. urticae infesting rose were carried out during the 2015 to 2016) at poly house, Department of Floriculture and Landscaping, ASPEE College of Horticulture and Forestry, N. A. U., Navsari. The observations on the incidence of spider mite were recorded at weekly interval, beginning from January 2015 and continued up to December 2016 on rose cv. Top secret. For sampling, three random leaves representing top, middle and bottom canopy were plucked from each of twenty five randomly selected plants per bed.

These leaves were held in separate properly labeled polyethylene bags and brought to the laboratory for numerical mite counts (live) from whole leaf under stereobinocular microscope. The data were recorded separately considering canopy and leaf surface for different standing rose plants. The observation on spider mite counts were recorded for two years in case of each standing rose plants under poly house condition. The data thus obtained were summed up and converted to total population per leaf (irrespective of plant canopy and leaf surface). To understand the pattern of distribution of spider mites on the plant and their preference to leaf surface, the data recorded on spider mite counts were summed up separately considering plant canopy.

\section{Results and Discussion}

The data recorded on the seasonal incidence of spider mite, $T$. urticae were presented and discussed yearwise as well as pooled over of two years.

\section{Year 2015}

It is evident from table 1 that the population of spider mite $T$. urticae remains active throughout the year under poly house condition. The mean mite population during $1^{\text {st }}$ standard meteorological week (SMW) i.e. first week of January was 0.07 mites per leaf. The average temperature and relative humidity of poly house during this period were $22.27^{\circ} \mathrm{C}$ and 59.52 per cent, respectively. The spider mite population on rose $\mathrm{cv}$. top secret under poly house remains active round the year and gradually increased during various months of year. It increased at faster rate and reached to its peak during the $31^{\text {st }}$ SMW i.e. first week of August 2015 with 11.99 spider mites per leaf. The average temperature and relative humidity of poly house during this period were $29.65^{\circ} \mathrm{C}$ and 63.70 per cent, respectively. The spider mite population then it has started declining in the later weeks of the year.The average population of spider mite, T. urticae was 5.11 per leaf. The distribution of spider mite, $T$. urticae on rose grown under poly house condition was also presented in table 1 . Throughout the crop season the population of T. urticae remains higher on the top leaves (9.77 mites per leaf), followed by middle leaves (4.22 mites per leaf) and lowest population were recorded on bottom leaf canopy (1.34 mites per leaf).

The correlation between $T$. urticae and abiotic factors like maximum temperature, minimum temperature, average temperature and average relative humidity of poly house were also worked out. It is clearly seen from table 3 that 
there were a highly significant positive correlation between population of $T$. urticae with maximum temperature $(\mathrm{r}=0.379)$, minimum temperature $(\mathrm{r}=0.560)$ and average temperature $(\mathrm{r}=0.512)$. The average relative humidity at the time of observation had a nonsignificant negative correlation $(\mathrm{r}=-0.060)$ with spider mite population.

\section{Year 2016}

It is evident from table 1 that the population of spider mite $T$. urticae remains active round the year under poly house condition. The mean mite population during $1^{\text {st }}$ standard meteorological week (SMW) i.e. first week of January was 0.33 mite per leaf. The average temperature and relative humidity of poly house during this period were $24.06^{\circ} \mathrm{C}$ and 69.85 per cent, respectively.

The spider mite population under poly house remains active round the year and gradually increased during various months of year. It increased at faster rate and reached to its peak during the $29^{\text {th }}$ SMW i.e. third week of July 2016 with 12.47 spider mites per leaf. The average temperature and relative humidity was $32.80^{\circ} \mathrm{C}$ and 84.78 per cent, respectively. Then the spider mite population has started declining in the later weeks of the year.The average population of the T. urticae was 5.27 mites per leaf. The distribution of $T$. urticae on rose grown under poly house condition was also presented in table 1.

Throughout the crop season the population of spider mite $T$. urticae remains higher on the top leaves (10.37 mites per leaf), followed by middle leaves (4.16 per mites leaf) and lowest population were recorded on bottom leaf canopy (1.27 mites per leaf).

The correlation between spider mite $T$. urticae and abiotic factors of poly house viz., maximum temperature, minimum temperature, average temperature and average relative humidity of poly house were also worked out. It is clearly seen from table 3 that there were a highly significant positive correlation between population of spider mite $T$. urticae with minimum temperature $(\mathrm{r}=0.866)$, average temperature $(\mathrm{r}=0.647)$ and average relative humidity $(\mathrm{r}=0.740)$. The maximum temperature had also a significant positive correlation $(r=0.278)$ with spider mite $T$. urticae population on rose.

\section{Pooled}

It is evident from table 2 that the population of spider mite $T$. urticae remains active round the year under poly house condition. The mean mite population during $1^{\text {st }}$ standard meteorological week (SMW) i.e. first week of January was 0.20 mite per leaf. The average temperature and relative humidity of poly house during this period were $23.16^{\circ} \mathrm{C}$ and 64.69 per cent, respectively.

The spider mite population under poly house remains active round the year and gradually increased during various months of year. It increased at faster rate and reached to its peak during the $29^{\text {th }}$ SMW with 11.77 spider mites per leaf. The average temperature and relative humidity was $31.78^{\circ} \mathrm{C}$ and 72.04 per cent, respectively.

Then the spider mite population has started declining in the later weeks of the year. The average population of the T. urticae was 5.19 mites per leaf. The distribution of T. urticae on rose grown under poly house condition was also presented in table 2 .

Throughout the crop season the population of spider mite $T$. urticae remains higher on the top leaves (10.07 mites per leaf), followed by middle leaves (4.19 per mites leaf) and lowest population were recorded on bottom leaf canopy (1.31 mites per leaf). 
Table.1 Seasonal incidence of spider mite, Tetranychus urticae on rose (cv. Top secret) under poly house condition

\begin{tabular}{|c|c|c|c|c|c|c|c|c|c|c|c|c|c|c|c|c|}
\hline \multirow{3}{*}{ SMW } & \multicolumn{8}{|c|}{ Mean number of spider mite per leaf } & \multicolumn{6}{|c|}{ Temperature $\left({ }^{\circ} \mathrm{C}\right)$} & \multicolumn{2}{|c|}{$\begin{array}{c}\text { Average } \\
\text { RH\% }\end{array}$} \\
\hline & \multicolumn{4}{|c|}{ Year 2015} & \multicolumn{4}{|c|}{ Year 2016} & \multicolumn{3}{|c|}{ Year 2015} & \multicolumn{3}{|c|}{ Year 2016} & \multirow{2}{*}{$\begin{array}{l}\text { Year } \\
2015\end{array}$} & \multirow{2}{*}{$\begin{array}{l}\text { Year } \\
2016\end{array}$} \\
\hline & Top & Middle & Bottom & Average & Top & Middle & Bottom & Average & Max. & Min. & Ave. & Max. & Min. & Ave. & & \\
\hline 1 & 0.20 & 0.00 & 0.00 & $\mathbf{0 . 0 7}$ & 0.80 & 0.20 & 0.00 & $\mathbf{0 . 3 3}$ & 30.33 & 14.20 & 22.27 & 33.91 & 14.20 & 24.06 & 59.52 & 69.85 \\
\hline 2 & 0.80 & 0.20 & 0.00 & $\mathbf{0 . 3 3}$ & 1.20 & 0.60 & 0.00 & 0.60 & 32.34 & 15.40 & 23.87 & 32.70 & 11.69 & 22.19 & 62.57 & 63.70 \\
\hline 3 & 1.00 & 0.64 & 0.12 & 0.59 & 1.40 & 1.00 & 0.00 & 0.80 & 31.04 & 13.70 & 22.37 & 28.93 & 10.47 & 19.70 & 66.54 & 53.95 \\
\hline 4 & 1.12 & 0.80 & 0.20 & 0.71 & 1.84 & 1.24 & 0.40 & 1.16 & 29.41 & 15.00 & 22.21 & 30.89 & 12.70 & 21.79 & 71.82 & 65.00 \\
\hline 5 & 1.48 & 1.12 & 0.28 & 0.96 & 2.20 & 1.48 & 0.60 & 1.43 & 31.24 & 20.60 & 25.92 & 32.61 & 15.00 & 23.81 & 71.90 & 65.75 \\
\hline 6 & 1.80 & 1.24 & 0.40 & 1.15 & 2.44 & 1.80 & 0.40 & 1.55 & 33.37 & 18.10 & 25.74 & 31.09 & 13.50 & 22.29 & 82.01 & 55.45 \\
\hline 7 & 2.12 & 1.60 & 0.48 & 1.40 & 2.40 & 2.00 & 0.80 & 1.73 & 33.83 & 19.90 & 26.86 & 30.27 & 13.20 & 21.74 & 84.08 & 56.50 \\
\hline 8 & 2.40 & 1.84 & 0.60 & 1.61 & 2.60 & 2.12 & 0.92 & 1.88 & 32.13 & 17.80 & 24.97 & 33.44 & 15.10 & 24.27 & 83.54 & 62.90 \\
\hline 9 & 2.64 & 2.00 & 0.72 & 1.79 & 2.68 & 1.92 & 1.00 & 1.87 & 32.02 & 18.90 & 25.46 & 35.09 & 17.80 & 26.44 & 81.70 & 68.55 \\
\hline 10 & 2.84 & 2.12 & 0.80 & 1.92 & 2.80 & 2.20 & 0.80 & 1.93 & 34.11 & 21.60 & 27.86 & 34.69 & 16.64 & 25.66 & 67.10 & 70.95 \\
\hline 11 & 3.00 & 2.40 & 0.84 & 2.08 & 3.00 & 2.60 & 0.80 & 2.13 & 33.91 & 23.80 & 28.86 & 34.30 & 17.61 & 25.96 & 66.60 & 82.35 \\
\hline 12 & 3.40 & 2.68 & 0.80 & 2.29 & 3.20 & 2.60 & 0.88 & 2.23 & 34.54 & 27.00 & 30.77 & 37.13 & 18.17 & 27.65 & 64.05 & 85.20 \\
\hline 13 & 3.88 & 2.80 & 0.92 & 2.53 & 3.48 & 2.84 & 1.00 & 2.44 & 36.69 & 24.60 & 30.64 & 38.83 & 18.76 & 28.79 & 59.45 & 83.15 \\
\hline 14 & 4.32 & 2.92 & 1.00 & 2.75 & 3.80 & 3.00 & 1.12 & 2.64 & 37.50 & 25.60 & 31.55 & 35.61 & 20.67 & 28.14 & 57.59 & 62.00 \\
\hline 15 & 4.60 & 3.00 & 1.08 & 2.89 & 4.20 & 3.16 & 1.20 & 2.85 & 35.90 & 26.30 & 31.10 & 37.61 & 20.23 & 28.92 & 56.40 & 62.50 \\
\hline 16 & 5.00 & 3.20 & 1.20 & 3.13 & 5.60 & 3.20 & 1.20 & $\mathbf{3 . 3 3}$ & 38.11 & 29.60 & 33.86 & 37.33 & 22.06 & 29.69 & 51.00 & 55.55 \\
\hline 17 & 5.80 & 3.36 & 1.16 & 3.44 & 6.00 & 3.60 & 1.40 & 3.67 & 38.80 & 31.20 & 35.00 & 34.60 & 22.04 & 28.32 & 51.60 & 56.44 \\
\hline 18 & 7.00 & 3.48 & 1.40 & 3.96 & 8.20 & 4.20 & 1.52 & 4.64 & 40.10 & 29.40 & 34.75 & 40.70 & 23.26 & 31.98 & 48.85 & 68.88 \\
\hline 19 & 8.20 & 3.64 & 1.60 & 4.48 & 10.40 & 4.00 & 1.60 & 5.33 & 37.83 & 28.30 & 33.06 & 38.40 & 26.00 & 32.20 & 46.31 & 72.11 \\
\hline 20 & 9.00 & 3.92 & 1.80 & 4.91 & 11.24 & 4.12 & 1.80 & 5.72 & 40.60 & 31.80 & 36.20 & 40.90 & 28.60 & 34.75 & 48.56 & 69.75 \\
\hline 21 & 11.24 & 4.20 & 1.92 & 5.79 & 13.12 & 5.00 & 1.80 & 6.64 & 43.90 & 32.20 & 38.05 & 44.30 & 30.90 & 37.60 & 46.65 & 69.47 \\
\hline 22 & 14.40 & 4.60 & 2.00 & 7.00 & 15.60 & 5.40 & 1.92 & 7.64 & 44.50 & 33.70 & 39.10 & 46.40 & 27.26 & 36.83 & 50.46 & 65.97 \\
\hline 23 & 15.60 & 5.00 & 2.24 & 7.61 & 16.80 & 6.00 & 2.00 & 8.27 & 44.20 & 34.60 & 39.40 & 45.50 & 30.00 & 37.75 & 52.15 & 79.30 \\
\hline 24 & 17.00 & 6.12 & 2.40 & 8.51 & 18.24 & 7.12 & 2.20 & 9.19 & 44.90 & 33.80 & 39.35 & 44.30 & 27.54 & 35.92 & 45.85 & 74.63 \\
\hline
\end{tabular}




\begin{tabular}{|c|c|c|c|c|c|c|c|c|c|c|c|c|c|c|c|c|}
\hline 25 & 18.80 & 7.00 & 2.60 & 9.47 & 19.80 & 9.00 & 2.44 & 10.41 & 43.50 & 32.20 & 37.85 & 44.50 & 31.90 & 38.20 & 51.70 & 71.91 \\
\hline 26 & 19.60 & 7.80 & 2.84 & 10.08 & 21.00 & 9.60 & 2.80 & 11.13 & 41.70 & 29.30 & 35.50 & 43.00 & 30.20 & 36.60 & 49.40 & 84.41 \\
\hline 27 & 20.48 & 8.20 & 2.92 & 10.53 & 23.40 & 8.80 & 2.80 & 11.67 & 40.30 & 26.61 & 33.46 & 41.90 & 31.70 & 36.80 & 52.16 & 88.36 \\
\hline 28 & 21.00 & 8.48 & 3.12 & 10.87 & 24.28 & 9.00 & 2.64 & 11.97 & 38.60 & 26.14 & 32.37 & 39.00 & 29.40 & 34.20 & 56.06 & 86.22 \\
\hline 29 & 21.60 & 8.60 & 3.00 & 11.07 & 25.60 & 9.20 & 2.60 & 12.47 & 36.30 & 25.23 & 30.76 & 36.70 & 28.90 & 32.80 & 59.30 & 84.78 \\
\hline 30 & 22.40 & 9.00 & 3.00 & 11.47 & 23.00 & 8.60 & 2.60 & 11.40 & 34.80 & 23.60 & 29.20 & 33.00 & 26.50 & 29.75 & 59.60 & 88.22 \\
\hline 31 & 23.60 & 9.40 & 2.96 & 11.99 & 21.80 & 8.12 & 2.40 & 10.77 & 35.50 & 23.80 & 29.65 & 30.90 & 25.50 & 28.20 & 63.70 & 91.57 \\
\hline 32 & 21.40 & 9.20 & 2.88 & 11.16 & 20.12 & 7.60 & 2.52 & 10.08 & 34.20 & 24.34 & 29.27 & 29.99 & 23.66 & 26.82 & 63.10 & 90.11 \\
\hline 33 & 20.00 & 8.20 & 2.60 & 10.27 & 18.92 & 6.80 & 2.20 & 9.31 & 31.69 & 24.50 & 28.09 & 30.97 & 24.66 & 27.81 & 62.23 & 82.89 \\
\hline 34 & 19.48 & 7.80 & 2.40 & 9.89 & 18.20 & 6.64 & 2.00 & 8.95 & 32.26 & 24.56 & 28.41 & 30.67 & 24.17 & 27.42 & 65.06 & 82.27 \\
\hline 35 & 18.00 & 7.40 & 2.16 & 9.19 & 17.80 & 7.00 & 2.00 & 8.93 & 32.76 & 23.76 & 28.26 & 31.17 & 24.01 & 27.59 & 70.58 & 81.12 \\
\hline 36 & 17.20 & 7.00 & 2.00 & 8.73 & 19.00 & 6.80 & 1.80 & 9.20 & 33.83 & 22.41 & 28.12 & 30.91 & 23.09 & 27.00 & 74.14 & 79.72 \\
\hline 37 & 16.60 & 6.76 & 1.84 & 8.40 & 18.12 & 6.20 & 1.64 & 8.65 & 32.64 & 22.69 & 27.66 & 31.07 & 22.76 & 26.91 & 85.93 & 81.87 \\
\hline 38 & 15.12 & 6.80 & 1.80 & 7.91 & 17.00 & 5.52 & 1.48 & 8.00 & 29.91 & 23.21 & 26.56 & 29.53 & 22.79 & 26.16 & 83.84 & 92.32 \\
\hline 39 & 14.84 & 6.68 & 1.60 & 7.71 & 16.52 & 5.16 & 1.40 & 7.69 & 33.36 & 21.87 & 27.61 & 31.91 & 22.33 & 27.12 & 75.24 & 83.92 \\
\hline 40 & 14.20 & 6.20 & 1.60 & 7.33 & 15.12 & 4.60 & 1.28 & 7.00 & 36.51 & 23.80 & 30.16 & 30.59 & 23.10 & 26.84 & 71.17 & 88.49 \\
\hline 41 & 13.00 & 5.80 & 1.36 & 6.72 & 13.60 & 4.00 & 1.20 & 6.27 & 36.10 & 23.26 & 29.68 & 31.19 & 21.90 & 26.54 & 76.41 & 83.22 \\
\hline 42 & 11.60 & 5.16 & 1.20 & 5.99 & 12.00 & 3.48 & 1.00 & 5.49 & 38.93 & 21.76 & 30.34 & 34.33 & 19.06 & 26.69 & 61.13 & 69.00 \\
\hline 43 & 10.40 & 5.00 & 1.08 & 5.49 & 10.80 & 3.60 & 0.92 & 5.11 & 36.90 & 21.37 & 29.14 & 33.86 & 17.47 & 25.66 & 66.26 & 59.96 \\
\hline 44 & 9.44 & 4.60 & 0.92 & 4.99 & 10.00 & 3.40 & 0.80 & 4.73 & 35.47 & 19.93 & 27.70 & 32.69 & 16.43 & 24.56 & 61.80 & 62.10 \\
\hline 45 & 8.40 & 4.00 & 0.80 & 4.40 & 8.60 & 3.00 & 0.64 & 4.08 & 36.29 & 19.06 & 27.67 & 34.26 & 13.70 & 23.98 & 58.87 & 50.81 \\
\hline 46 & 7.20 & 3.40 & 0.60 & 3.73 & 7.20 & 2.56 & 0.48 & 3.41 & 36.19 & 19.73 & 27.96 & 33.97 & 15.14 & 24.56 & 60.88 & 52.61 \\
\hline 47 & 5.80 & 2.00 & 0.40 & 2.73 & 5.44 & 2.12 & 0.36 & 2.64 & 34.97 & 20.81 & 27.89 & 33.94 & 12.57 & 23.26 & 60.36 & 45.52 \\
\hline 48 & 4.00 & 1.24 & 0.16 & 1.80 & 4.60 & 1.80 & 0.28 & 2.23 & 34.69 & 17.81 & 26.25 & 34.49 & 12.34 & 23.41 & 57.91 & 51.40 \\
\hline 49 & 2.40 & 0.60 & 0.08 & 1.03 & 2.00 & 1.36 & 0.20 & 1.19 & 35.00 & 14.07 & 24.54 & 33.41 & 14.44 & 23.93 & 54.48 & 49.58 \\
\hline 50 & 1.00 & 0.36 & 0.00 & 0.45 & 1.20 & 0.80 & 0.20 & 0.73 & 31.50 & 11.47 & 21.49 & 32.84 & 12.49 & 22.66 & 59.10 & 48.45 \\
\hline 51 & 0.80 & 0.08 & 0.00 & 0.29 & 0.80 & 0.12 & 0.00 & 0.31 & 31.13 & 10.47 & 20.80 & 32.86 & 13.67 & 23.26 & 49.15 & 48.57 \\
\hline 52 & 0.92 & 0.00 & 0.00 & 0.31 & 0.20 & 0.00 & 0.00 & $\mathbf{0 . 0 7}$ & 32.31 & 11.44 & 21.88 & 32.31 & 11.43 & 21.87 & 44.04 & 48.79 \\
\hline Mean & 9.77 & 4.22 & 1.34 & 5.11 & 10.37 & 4.16 & 1.27 & 5.27 & & & & & & & & \\
\hline
\end{tabular}


Table.2 Seasonal incidence of spider mite, Tetranychus urticae on rose (cv. Top secret) under poly house condition (Pooled)

\begin{tabular}{|c|c|c|c|c|c|c|c|c|}
\hline \multirow{2}{*}{ SMW } & \multicolumn{4}{|c|}{ Mean number of spider mite per leaf } & \multicolumn{3}{|c|}{ Temperature $\left({ }^{\circ} \mathrm{C}\right)$} & \multirow{2}{*}{$\begin{array}{c}\text { Average } \\
\text { RH\% }\end{array}$} \\
\hline & Top & Middle & Bottom & Average. & Max. & Min. & Ave. & \\
\hline 1 & 0.50 & 0.10 & 0.00 & 0.20 & 32.12 & 14.20 & 23.16 & 64.69 \\
\hline 2 & 1.00 & 0.40 & 0.00 & 0.47 & 32.52 & 13.54 & 23.03 & 63.13 \\
\hline 3 & 1.20 & 0.82 & 0.06 & 0.69 & 29.99 & 12.09 & 21.04 & 60.24 \\
\hline 4 & 1.48 & 1.02 & 0.30 & $\mathbf{0 . 9 3}$ & 30.15 & 13.85 & 22.00 & 68.41 \\
\hline 5 & 1.84 & 1.30 & 0.44 & 1.19 & 31.93 & 17.80 & 24.86 & 68.83 \\
\hline 6 & 2.12 & 1.52 & 0.40 & 1.35 & 32.23 & 15.80 & 24.01 & 68.73 \\
\hline 7 & 2.26 & 1.80 & 0.64 & 1.57 & 32.05 & 16.55 & 24.30 & 70.29 \\
\hline 8 & 2.50 & 1.98 & 0.76 & 1.75 & 32.79 & 16.45 & 24.62 & 73.22 \\
\hline 9 & 2.66 & 1.96 & 0.86 & 1.83 & 33.55 & 18.35 & 25.95 & 75.13 \\
\hline 10 & 2.82 & 2.16 & 0.80 & 1.93 & 34.40 & 19.12 & 26.76 & 69.03 \\
\hline 11 & 3.00 & 2.50 & 0.82 & 2.11 & 34.11 & 20.71 & 27.41 & 74.48 \\
\hline 12 & 3.30 & 2.64 & 0.84 & 2.26 & 35.84 & 22.59 & 29.21 & 74.63 \\
\hline 13 & 3.68 & 2.82 & 0.96 & 2.49 & 37.76 & 21.68 & 29.72 & 71.30 \\
\hline 14 & 4.06 & 2.96 & 1.06 & 2.69 & 36.56 & 23.14 & 29.85 & 59.80 \\
\hline 15 & 4.40 & 3.08 & 1.14 & 2.87 & 36.76 & 23.26 & 30.01 & 59.45 \\
\hline 16 & 5.30 & 3.20 & 1.20 & 3.23 & 37.72 & 25.83 & 31.78 & 53.28 \\
\hline 17 & 5.90 & 3.48 & 1.28 & 3.55 & 36.70 & 26.62 & 31.66 & 54.02 \\
\hline 18 & 7.60 & 3.84 & 1.46 & 4.30 & 40.40 & 26.33 & 33.36 & 58.87 \\
\hline 19 & 9.30 & 3.82 & 1.60 & 4.91 & 38.11 & 27.15 & 32.63 & 59.21 \\
\hline 20 & 10.12 & 4.02 & 1.80 & 5.31 & 40.75 & 30.20 & 35.48 & 59.16 \\
\hline 21 & 12.18 & 4.60 & 1.86 & 6.21 & 44.10 & 31.55 & 37.83 & 58.06 \\
\hline 22 & 15.00 & 5.00 & 1.96 & 7.32 & 45.45 & 30.48 & 37.96 & 58.21 \\
\hline 23 & 16.20 & 5.50 & 2.12 & 7.94 & 44.85 & 32.30 & 38.58 & 65.73 \\
\hline 24 & 17.62 & 6.62 & 2.30 & 8.85 & 44.60 & 30.67 & 37.64 & 60.24 \\
\hline 25 & 19.30 & 8.00 & 2.52 & 9.94 & 44.00 & 32.05 & 38.03 & 61.81 \\
\hline 26 & 20.30 & 8.70 & 2.82 & 10.61 & 42.35 & 29.75 & 36.05 & 66.90 \\
\hline 27 & 21.94 & 8.50 & 2.86 & 11.10 & 41.10 & 29.16 & 35.13 & 70.26 \\
\hline 28 & 22.64 & 8.74 & 2.88 & 11.42 & 38.80 & 27.77 & 33.29 & 71.14 \\
\hline 29 & 23.60 & 8.90 & 2.80 & 11.77 & 36.50 & 27.06 & 31.78 & 72.04 \\
\hline 30 & 22.70 & 8.80 & 2.80 & 11.43 & 33.90 & 25.05 & 29.48 & 73.91 \\
\hline 31 & 22.70 & 8.76 & 2.68 & 11.38 & 33.20 & 24.65 & 28.93 & 77.63 \\
\hline
\end{tabular}




\begin{tabular}{|l|c|c|c|c|c|c|c|c|}
\hline $\mathbf{3 2}$ & 20.76 & 8.40 & 2.70 & $\mathbf{1 0 . 6 2}$ & 32.09 & 24.00 & 28.05 & 76.60 \\
\hline $\mathbf{3 3}$ & 19.46 & 7.50 & 2.40 & $\mathbf{9 . 7 9}$ & 31.33 & 24.58 & 27.95 & 72.56 \\
\hline $\mathbf{3 4}$ & 18.84 & 7.22 & 2.20 & $\mathbf{9 . 4 2}$ & 31.46 & 24.36 & 27.91 & 73.66 \\
\hline $\mathbf{3 5}$ & 17.90 & 7.20 & 2.08 & $\mathbf{9 . 0 6}$ & 31.96 & 23.89 & 27.93 & 75.85 \\
\hline $\mathbf{3 6}$ & 18.10 & 6.90 & 1.90 & $\mathbf{8 . 9 7}$ & 32.37 & 22.75 & 27.56 & 76.93 \\
\hline $\mathbf{3 7}$ & 17.36 & 6.48 & 1.74 & $\mathbf{8 . 5 3}$ & 31.86 & 22.72 & 27.29 & 83.90 \\
\hline $\mathbf{3 8}$ & 16.06 & 6.16 & 1.64 & $\mathbf{7 . 9 5}$ & 29.72 & 23.00 & 26.36 & 88.08 \\
\hline $\mathbf{3 9}$ & 15.68 & 5.92 & 1.50 & $\mathbf{7 . 7 0}$ & 32.64 & 22.10 & 27.37 & 79.58 \\
\hline $\mathbf{4 0}$ & 14.66 & 5.40 & 1.44 & $\mathbf{7 . 1 7}$ & 33.55 & 23.45 & 28.50 & 79.83 \\
\hline $\mathbf{4 1}$ & 13.30 & 4.90 & 1.28 & $\mathbf{6 . 4 9}$ & 33.64 & 22.58 & 28.11 & 79.82 \\
\hline $\mathbf{4 2}$ & 11.80 & 4.32 & 1.10 & $\mathbf{5 . 7 4}$ & 36.63 & 20.41 & 28.52 & 65.07 \\
\hline $\mathbf{4 3}$ & 10.60 & 4.30 & 1.00 & $\mathbf{5 . 3 0}$ & 35.38 & 19.42 & 27.40 & 63.11 \\
\hline $\mathbf{4 4}$ & 9.72 & 4.00 & 0.86 & $\mathbf{4 . 8 6}$ & 34.08 & 18.18 & 26.13 & 61.95 \\
\hline $\mathbf{4 5}$ & 8.50 & 3.50 & 0.72 & $\mathbf{4 . 2 4}$ & 35.27 & 16.38 & 25.83 & 54.84 \\
\hline $\mathbf{4 6}$ & 7.20 & 2.98 & 0.54 & $\mathbf{3 . 5 7}$ & 35.08 & 17.44 & 26.26 & 56.74 \\
\hline $\mathbf{4 7}$ & 5.62 & 2.06 & 0.38 & $\mathbf{2 . 6 9}$ & 34.46 & 16.69 & 25.58 & 52.94 \\
\hline $\mathbf{4 8}$ & 4.30 & 1.52 & 0.22 & $\mathbf{2 . 0 1}$ & 34.59 & 15.08 & 24.83 & 54.66 \\
\hline $\mathbf{4 9}$ & 2.20 & 0.98 & 0.14 & $\mathbf{1 . 1 1}$ & 34.21 & 14.26 & 24.23 & 52.03 \\
\hline $\mathbf{5 0}$ & 1.10 & 0.58 & 0.10 & $\mathbf{0 . 5 9}$ & 32.17 & 11.98 & 22.08 & 53.77 \\
\hline $\mathbf{5 1}$ & 0.80 & 0.10 & 0.00 & $\mathbf{0 . 3 0}$ & 31.99 & 12.07 & 22.03 & 48.86 \\
\hline $\mathbf{5 2}$ & 0.56 & 0.00 & 0.00 & $\mathbf{0 . 1 9}$ & 32.31 & 11.43 & 21.87 & 46.41 \\
\hline $\mathbf{M e a n}$ & 10.07 & 4.19 & 1.31 & $\mathbf{5 . 1 9}$ & & & & \\
\hline SMW & Stand ard & Meteorologic al Week & & & &
\end{tabular}

Table.3 Correlation of two spotted red spider mite, T. urticae Koch with the abiotic factors of poly house on rose (cv. Top secret)

\begin{tabular}{|l|c|c|c|}
\hline Abiotic parameters & Year 2015 & Year 2016 & Pooled \\
\hline Maximum Temperature $\left.\mathbf{(}^{\mathbf{0}} \mathbf{C}\right)$ & $0.379^{* *}$ & $0.278^{*}$ & $0.327^{*}$ \\
\hline Minimum Temperature $\left({ }^{\mathbf{0}} \mathbf{C}\right)$ & $0.560^{* *}$ & $0.866^{* *}$ & $0.748^{* *}$ \\
\hline Average Temperature $\mathbf{(}^{\mathbf{C}} \mathbf{C}$ & $0.512^{* *}$ & $0.687^{* *}$ & $0.611^{* *}$ \\
\hline Average RH \% & -0.060 & $0.740^{* *}$ & $0.486^{* *}$ \\
\hline *Significant 5\%
\end{tabular}

*Significant at $5 \%$ level of significance

**Highly significant at $1 \%$ level of significance 
The correlation between spider mite T. urticae and abiotic factors of poly house viz., maximum temperature, minimum temperature, average temperature and average relative humidity of poly house were also worked out. It is clearly seen from table 3 that there were a highly significant positive correlation between population of spider mite $T$. urticae with minimum temperature $(\mathrm{r}=0.748)$, average temperature $(\mathrm{r}=0.611)$ and average relative humidity $(\mathrm{r}=0.486)$. The maximum temperature had also a significant positive correlation $(\mathrm{r}=0.327)$ with spider mite $T$. urticae population on rose.

Sudhirkumar and Shelke (2008) reported that the spider mite population started from $35^{\text {th }}$ SMW with $1^{\text {st }}$ peak at $42^{\text {nd }}$ SMW and reached up to lowest population during $1^{\text {st }}$ SMW. Whereas, pest population also started from $2^{\text {nd }}$ SMW and reached its $2^{\text {nd }}$ peak at $11^{\text {th }}$ SMW. Dhar et al., (2000) found $30{ }^{\circ} \mathrm{C}$ temperature and 78.5 per cent relative humidity to be optimum for highest incidence of $T$. urticae (both eggs and mobile stages) on okra. Recently, Shah (2014) from Navsari also studied the seasonal activities of spider mite T. urticae under poly house gerbera and found that the eggs and mobile stages of T. urticae showed a non-significant negative correlation with temperature whereas, it had a significant positive correlation with relative humidity. It was also found that on gerbera the spider mite remains active throughout the crop season with a peak during $31^{\text {st }}$ SMW (last week of July), thus more or less in support of the present findings. Further, Mazid et al., (2015) from their studies on Oligonychus coffeae Nietner concluded that there was a significant positive correlation of the population of spider mites with the increasing temperature whereas moderate positive correlation existed between relative humidity. Further, the distribution of spider mite on the plant canopy was also recorded and it was found that maximum activities of $T$. urticae were recorded on top canopy followed by middle and bottom canopy. It was due to the continuous supply of vital nutrients which are nutritive for spider mite. In past, Shah (2014), Pokle and Shukla (2015) and Chauhan and Shukla (2016) also reported that the spider mite, $T$. urticae lays maximum eggs on top canopy of gerbera, tomato and French bean with maximum mobile stages.

\section{Acknowledgement}

The authors are thankful to the Professor and Head, Department of Entomology, N. M. College of Agriculture and The Principal, N. M. College of Agriculture, Navsari for providing necessary facilities during the present study. The authors are also thankful to the Director of Research and Dean, Post Graduate studies, Navsari Agricultural University, Navsari for their kind support and help during the study period.

\section{References}

Anonymous, 2016. National Horticulture Board, Gurgaon. Ministry of Agriculture, Government of India.

Chauhan, R., and Shukla, A. 2016. Population dynamics of two spotted spider mite, Tetranychus urticae Koch on French bean (Phaseolus vulgaris L.). Inter. J. of Pl. Pro, 9(2): 536-539.

Dhar, T., Dey, P. K. and Sarkar, P. K. 2000. Influence of abiotic factors on population build-up of red spider mite Tetranychus urticae on okra vis-à-vis evaluation of some new pesticides for their control. Pestology, 24(9): 34-37.

Mazid, S., Rajkhowa, R. C. and Kalita, J. C. 2015. Seasonal incidence of red spider mite, Oligonychus coffeae Nietner on tea plantation in Assam. Indian Journal of Research, 4(3): 6-7.

Pokle, P.P., and Shukla, A. 2015. Population dynamics of two spotted spider mite, 
Tetranychus urticae (Koch) (Acari: Tetranychidae) on tomato under polyhouse condition. J. Appl. Biosci., 41(2): 148-151.

Shah, D. R., 2014. Study on mite (Tetranychus urticae Koch.) infesting gerbera (Gerbera jamesonii) under polyhouse condition. M.Sc. (Agri.)
Thesis submitted to Navsari Agricultural University, Navsari.

Sudhirkumar, S., and Shelke, S. S. 2008. Seasonal incidence of two spotted spider mite (Tetranychus urticae Koch.) on rose. J. Maharashtra Agric. Univ., 33(3): 406-407.

\section{How to cite this article:}

Desai, S.R., K.G. Patel and Abhishek Shukla. 2017. Seasonal Incidence of Spider Mite (Tetranychus urticae Koch) (Acari: Tetranychidae) Infesting Rose under Poly House Condition. Int.J.Curr.Microbiol.App.Sci. 6(9): 2661-2669. doi: https://doi.org/10.20546/ijcmas.2017.609.328 